\title{
Weak Reflections in the Laves Phase $\mathrm{FeBe}_{2}$
}

\author{
M. Margarida R. Costa, Maria José B. M. de Almeida, and José António Paixão \\ Departamento de Física - Centro FC1 INIC - Universidade de Coimbra, 3000 Coimbra, Portugal
}

Z. Naturforsch. 48a, 47-50 (1993); received December 6, 1991

\begin{abstract}
X-ray diffraction intensities from a single crystal of the Laves phase $\mathrm{FeBe}_{2}$ were collected at room temperature. Atomic and thermal parameters as well as a scale factor were determined by leastsquares refinements of high-angle data $\left[(\sin \theta) / \lambda \geqq 0.6 \AA^{-1}\right]$.

The results of Fourier inversion of the data are presented in terms of maps of the total density and followed by a discussion of the "effects" of two criteria for labelling reflection intensities as "unobserved", based on their statistical accuracy.
\end{abstract}

Key words: Weak reflections, Laves phases, $\mathrm{FeBe}_{2}$.

\section{Introduction}

The present work is part of a project aiming at the investigation of the charge density of Laves-phase compounds $[1,2]$, in order to clarify the rôle of atomic size (the so-called "size-factor") and electron concentration in the formation of such compounds [3-5].

This contribution describes preliminary work on the Laves phase $\mathrm{FeBe}_{2}$, which has the $\mathrm{MgZn}_{2}(\mathrm{C} 14)$ hexagonal structure, with space group $\mathrm{P}_{3} / \mathrm{mmc}$. In particular it aims at discussing the choice of rejection criteria for "unobserved" reflection intensities on the basis of their statistical accuracy.

\section{Experimental}

A single crystal of $\mathrm{FeBe}_{2}$ with approximate dimensions $(0.08 \times 0.08 \times 0.15) \mathrm{mm}^{3}$, was selected from a bulk sample, kindly supplied by Dr. P. J. Brown (Institut Laue-Langevin, Grenoble, France).

Intensity measurements were carried out on a CAD4 four-circle diffractometer, using $\mathrm{MoK} \alpha$ radiation diffracted by the (002) planes of a graphite crystal $(\lambda=0.70926 \AA)$.

Lattice parameters were derived from the angular positions of 25 reflections, as

$$
\begin{aligned}
& a=b=4.2224(10) \AA, \quad c=6.8700(11) \AA, \\
& \alpha=\beta=90^{\circ}, \quad \gamma=120^{\circ} .
\end{aligned}
$$

\footnotetext{
* Presented at the Sagamore X Conference on Charge, Spin, and Momentum Densities, Konstanz, Fed. Rep. of Germany, September 1-7, 1991.

Reprint requests to Prof. Dr. M. Margarida R. Costa, Departamento de Física, Universidade de Coimbra, P-3000 Coimbra, Portugal.
}

All accessible reflections (6696) within an Ewald sphere of radius $1.22 \AA^{-1}$ were measured in $\omega-2 \theta$ scans. Five standard reflections were periodically measured to check the stability of the main beam.

\section{Data Analysis}

The analysis of the raw data was performed with SDP programs [6] using a MICROVAX II computer with VMS operating system.

In order to correct for intensity fluctuations in the direct beam, data sub-sets measured in between any two groups of standard reflections were re-scaled against the appropriate standards.

The usual Lorentz and polarization corrections were applied to all integrated intensities.

In order to avoid uncertainties in the description of the irregular shape and consequent estimation of the volume of the crystal, an absorption correction based on psi-scans of a few sets of equivalent reflections with $80^{\circ}<\chi<90^{\circ}$ was applied [7]. Maximum and minimum absorption corrections were 0.9985 and 0.7074 ; the agreement factor for the observed structure factors of equivalent reflections thus corrected was $3.2 \%$.

After averaging the intensities of the equivalent reflections, 441 independent reflections were obtained.

As a first attempt, all reflection intensities with $I_{h k l} \leqq 3 \sigma_{h k l}\left(\sigma_{h k l}\right.$ being the standard deviation of $\left.I_{h k l}\right)$ were considered as "unobserved". This criterion has been currently used by the authors in charge density studies of a number of materials, namely rutile-structure compounds.

The use of a less restrictive criterion for labelling "unobserved" reflections (e.g. $I_{h k l} \leqq \sigma_{h k l}$ ) has been

0932-0784 / 93 / 0100-0047 \$ 01.30/0. - Please order a reprint rather than making your own copy. 
Table 1.

\begin{tabular}{|c|c|c|}
\hline & $\begin{array}{l}I>\sigma \\
\text { Indep. refl. ..... } 335 \\
\text { Indep. refl. with } \\
\left(\frac{\sin \theta}{\lambda}\right) \geqq 0.6 \AA^{-1} 289\end{array}$ & $\begin{array}{l}I>3 \sigma \\
\text { Indep. refl. ..... } 254 \\
\text { Indep. refl. with } \\
\left(\frac{\sin \theta}{\lambda}\right) \geqq 0.6 \AA^{-1} 211\end{array}$ \\
\hline $\mathrm{Fe}(3 \mathrm{~m})$ & & \\
\hline$z$ & $0.06161(4)$ & $0.06168(5)$ \\
\hline$U_{11}=U_{22}$ & $0.00554(5)$ & $0.00537(6)$ \\
\hline$U_{12}^{11}$ & 0.00277 (3) & 0.00269 (3) \\
\hline$U_{33}^{12}$ & $0.00289(10)$ & $0.00282(14)$ \\
\hline $\begin{array}{l}\mathrm{Be}_{1}(\overline{3} \mathrm{~m}) \\
U_{11}=U_{22} \\
U_{12} \\
U_{33}\end{array}$ & $\begin{array}{l}0.01100(147) \\
0.00550(73) \\
0.00586(138)\end{array}$ & $\begin{array}{l}0.00855(142) \\
0.00478(71) \\
0.00961(165)\end{array}$ \\
\hline $\begin{array}{l}\mathrm{Be}_{\text {II }}(\mathrm{mm}) \\
x \\
U_{11} \\
U_{22} \\
U_{12} \\
U_{33}\end{array}$ & $\begin{array}{l}0.83031(26) \\
0.00808(77) \\
0.00756(110) \\
0.00378(55) \\
0.00521(62)\end{array}$ & $\begin{array}{l}0.83012(63) \\
0.01010(96) \\
0.00701(131) \\
0.00352(66) \\
0.00519(60)\end{array}$ \\
\hline $\begin{array}{l}S \\
g \\
R \\
R_{w}\end{array}$ & $\begin{array}{l}0.47433(192) \\
2.46(70) \times 10^{-6} \\
2.6 \% \\
3.3 \%\end{array}$ & $\begin{array}{l}0.47463(220) \\
2.05(73) \times 10^{-6} \\
3.0 \% \\
3.3 \%\end{array}$ \\
\hline
\end{tabular}

tested by the authors in a few cases; in general, the inclusion of weak intensities in the least-squares refinements does not yield any significant differences neither on the atomic and thermal parameters, nor on the difference density maps.

Although the rejection of intensities with $I_{h k l} \leqq$ $3 \sigma_{h k l}$ appears to be adequate in most cases, the influence of weak reflections (e.g., those with $\sigma_{h k l} \leqq I_{h k l}$ $\left.\leqq 3 \sigma_{h k l}\right)$ should always be assessed, prior to a final choice of rejection criteria.

The Laves phase $\mathrm{FeBe}_{2}$ can be used as an illustrative example of the above considerations.

In this structure the $\mathrm{Fe}$ atoms occupy positions with local symmetry $(3 \mathrm{~m})$, whereas the Be atoms can be found in positions with two distinct symmetries: $\overline{3} \mathrm{~m}$ $\left(\mathrm{Be}_{\mathrm{I}}\right)$ and $\mathrm{mm}\left(\mathrm{Be}_{\mathrm{II}}\right)$. The systematic absences originating in these local symmetries are such that only $2 \mathrm{Be}$ atoms in a total of 12 atoms in the unit cell ( 4 of which are $\mathrm{Fe}$ ) contribute to a class of reflections with indices such that $h-k=3 n$ and $l=2 n+1$. Taking into account the low atomic number of $\mathrm{Be}$ (as compared to $\mathrm{Fe}$ ) this implies that all these reflections are very weak and therefore will be labelled as "unobserved" under the requirement $I \leqq 3 \sigma$.

Therefore, a second data set including all reflection intensities with $I_{h k l} \geqq \sigma_{h k l}$ was selected for the subsequent least-squares analysis.

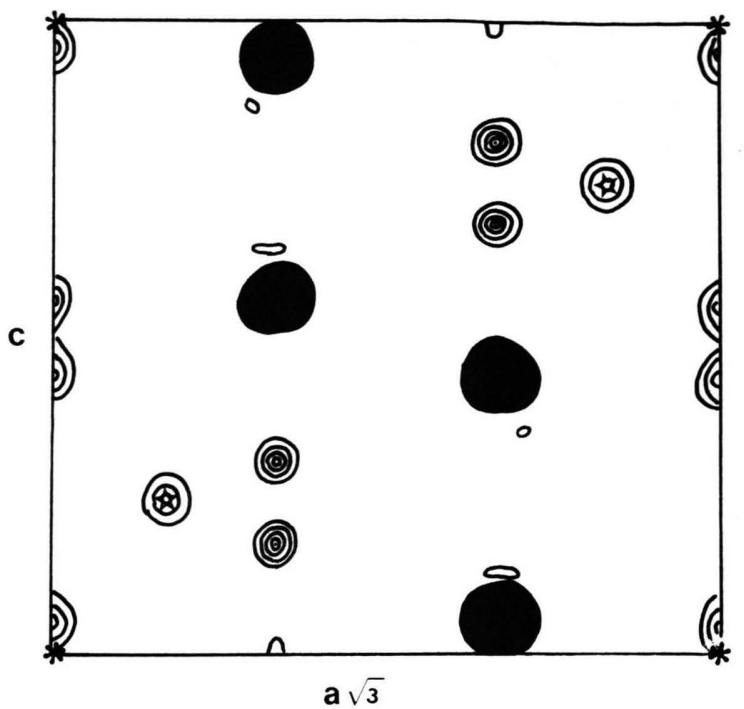

Fig. 1. Fourier maps $S F_{\text {obs }}$, for data set $I>3 \sigma$. Contour levels at intervals of $10 \mathrm{e}^{-3}$. Section [11.0] of the unit cell. - $\mathrm{Fe}$ atoms; $* \mathrm{Be}_{\mathrm{I}}$ atoms; is $\mathrm{Be}_{\mathrm{II}}$ atoms.

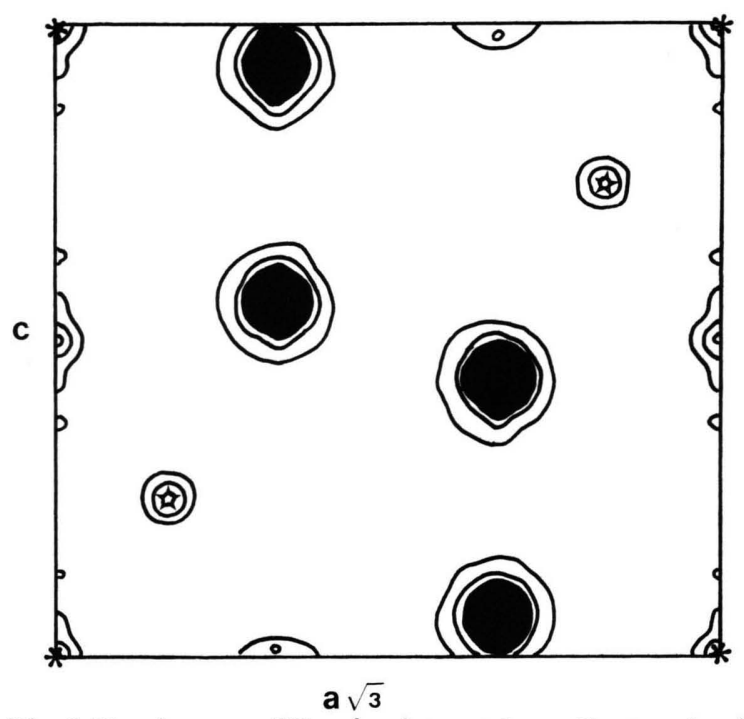

Fig. 2. Fourier maps $S F_{\text {obs }}$, for data set $I>\sigma$. Contour levels at invervals of $10 \mathrm{e}^{-3}$. Section [11.0] of the unit cell. - Fe atoms; $* \mathrm{Be}_{\mathrm{I}}$ atoms; is $\mathrm{Be}_{\mathrm{II}}$ atoms.

Structure factors $\left(F_{\text {calc }}\right)$ for observed reflections were calculated assuming spherical distributions of the atomic electrons. Atomic scattering factors with anomalous-dispersion corrections, both tabulated in the International Tables for X-Ray Crystallography [8], were used. 
Full-matrix least-squares refinements on $\left(\left|F_{\text {obs }}\right|\right.$ $\left.-\left|F_{\text {calc }}\right|\right)^{2}$ were performed on each data set, using a non-Poisson weighting scheme in which the weight of a reflection is calculated as $\omega=1 /\left(\sigma_{F}\right)^{2}$, where $\sigma_{F}=\left[\left(\sigma_{I}\right)^{2}+\left(p F^{2}\right)^{2}\right]^{1 / 2} / 2 F ; p$ is an instrumental factor used to downweight strong reflections; a value of 0.04 for $p$ was used in this work.

Positional and anisotropic thermal parameters $U_{i j}$ for the constituent atoms as well as a scale factor were refined from high-angle data $\left(\sin \theta / \lambda \geqq 0.6 \AA^{-1}\right)$. An extinction parameter $g$ was refined independently (i.e. after fixing all the above parameters at the refined values) are described in [9] and used to correct for extinction. All refinements were carried out until a shift/e.s.d. ratio smaller than 0.001 was achieved for all parameters refined.

The results of both refinements as well as other relevant information are shown on Table 1 . The overall agreement is verified within one standard deviation (or two in a few cases where the error is small). Only the $R$-factor is slightly higher, as expected, in the case where reflections with lower statistical accuracy $(\sigma \leqq I \leqq 3 \sigma)$ are included.

The effect of using the criterion $I \geqq 3 \sigma$, therefore excluding a particular class of weak reflections $(h-k$ $=3 n$ and $l=2 n+1)$ can, however, be clearly seen when Fourier inversion of the observed data is performed.

The results are easily visualised in terms of total density maps for different sections of the unit cell; the most relevant of these is shown in Fig. 1, where two striking features can be observed:

(i) the electron density around $\mathrm{Be}_{\mathrm{I}}$ atoms is not spherical but evidences two maxima symmetrically positioned with respect to the "atomic position".

(ii) a peak of electron density with approximate spherical symmetry appears below and above the center of each triangular arrangement of $\mathrm{Be}_{\mathrm{II}}$ atoms. Each of these can be seen to represent a significantly higher density than that observed at the $\mathrm{Be}_{\mathrm{II}}$ atomic positions.

This is a typical "spurious" effect simulating a higher symmetry, which is known to occur when a particular set of reflections is not included in the Fourier analysis.
These "anomalous" features disappear when the weak reflections $(I \geqq \sigma)$ are included, i.e., when Fourier inversion is performed on a complete data set (Figure 2).

\section{Conclusions}

From this preliminary study of the Laves phase $\mathrm{FeBe}_{2}$ it is noticeable that an effort has to be made in order to improve the agreement factors between equivalent reflections before a study of the electron density is achieved. In order to reduce the uncertainties introduced by any absorption correction, attempts will be made to select a single crystal with a more regular shape, ideally a sphere with appropriate dimensions.

However, the present results can be used to demonstrate the need for a critical analysis of the rejection criteria for "weak" reflections. The authors' experience tends to favour the $I>3 \sigma$ criterion on the basis of better data accuracy and little or no influence of weaker reflections in the electron density. It is advisable, though, to test this influence on each individual data set. In particular, the use of the above criterion may lead to the rejection of a class of reflections which, owing to the type of structure and to the nature of the atoms in the compound, are all weak. The use of such an incomplete data set has the mathematically understandable effect of leading to spurious features or symmetries in the Fourier maps, as illustrated in the present work.

\section{Acknowledgements}

We are indebted to the Cultural Service of the Embassy of the Federal Republic of Germany, the Deutscher Akademischer Austauschdienst (DAAD) and the German Agency for Technical Cooperation (GTZ) for the offer of a CAD4 automatic diffractometer, which enabled the experimental work to be carried out. 
[1] M. J. M. de Almeida, M. M. R. Costa, and J. A. Paixão, Portugal. Phys. 19, 289 (1988).

[2] A. M. Beja, L. Alte da Veiga, L. C. R. Andrade, M. M. R. Costa, and J. A. Paixão, Portugal. Phys. 19, 293 (1988).

[3] J. B. Friauf, J. Amer. Chem. Soc. 49, 3107 (1927).

[4] F. Laves and H. Witte, Metallwirtsch. Metallwiss. Metalltech. 14, 645 (1935)

[5] A. K. Sinha, Progress in Materials Science 15, 93 (1972).
[6] B. A. Frenz, Enraf-Nonius Structure Determination Package; SDP Users Guide, Enraf-Nonius, Delft, The Netherlands.

[7] A. C. T. North, D. C. Phillips, and F. S. Mathews, Acta Cryst. A 24, 351 (1968).

[8] International Tables for Crystallography (1974), Vol. IV.

[9] M. J. M. de Almeida, M. M. R. Costa, and J. A. Paixão, Acta Cryst. B 45, 549 (1989). 